\title{
452.
}

\section{ON AN ANALYTICAL THEOREM FROM A NEW POINT OF VIEW.}

[From the Proceedings of the London Mathematical Society, vol. III. (1869-1871), pp. 220, 221. Read February 9, 1871.]

THE theorem is a well-known one, derived from the equation

$$
\left(a z^{2}+2 b z+c\right) w^{2}+2\left(a^{\prime} z^{2}+2 b^{\prime} z+c^{\prime}\right) w+a^{\prime \prime} z^{2}+2 b^{\prime \prime} z+c^{\prime \prime}=0 ;
$$

viz., considering this equation as establishing a relation between the variables $z$ and $w$, and writing it in the forms

$$
2 u=A w^{2}+2 B w+C=A^{\prime} z^{2}+2 B^{\prime} z+C^{\prime}=0,
$$

(where, of course, $A, B, C$ are quadric functions of $z$, and $A^{\prime}, B^{\prime}, C^{\prime}$ quadric functions of $w$,) we have

$$
0=\frac{d u}{d w} d w+\frac{d u}{d z} d z,=(A w+B) d w+\left(A^{\prime} z+B^{\prime}\right) d z
$$

but in virtue of the equation $u=0$, we have $A w+B=\sqrt{B^{2}-A C}$, and $A^{\prime} z+B^{\prime}=\sqrt{B^{\prime 2}}-A^{\prime} C^{\prime}$, and the differential equation thus becomes

$$
\frac{d w}{\sqrt{B^{\prime 2}-A^{\prime} C^{\prime}}}+\frac{d z}{\sqrt{B^{2}-A C}}=0,
$$

where $B^{\prime 2}-A^{\prime} C^{\prime}$ and $B^{2}-A C$ are quartic functions of $w$ and $z$ respectively. This is, of course, integrable (viz., the integral is the original equation $u=0$ ); and it follows, from the theory of elliptic functions, that the two quartic functions must be linearly transformable into each other; viz., they must have the same absolute invariant $I^{3} \div J^{2}$. It is, in fact, easy to verify, not only that this is so, but that the two functions have the same quadrinvariant $I$, and the same cubinvariant $J$. 
The new point of view is, that we take the coefficients $a, b$, \&c., to be homogeneous finctions of $(x, y)$, their degrees being such that the equation $u=0$ is a quartic equation $(* \gamma x, y, z, w)^{4}=0$; viz., this equation now represents a quartic surface having a node (conical point) at the point $(x=0, y=0, z=0)$, and also a node at the point $(x=0, y=0, w=0)$, say, these points are $O, O^{\prime}$ respectively. The equation $B^{\prime 2}-A^{\prime} C^{\prime}=0$ gives the circumscribed sextic cone having $O$ for its vertex, and the equation $B^{2}-A C=0$ the circumscribed sextic cone having $O^{\prime}$ for its vertex; each of these cones has the line $O O^{\prime}(x=0, y=0)$ for a nodal line, as appears geometrically, and also by the equations containing $z, w$ respectively in the degree 4 . Considering $B^{\prime 2}-A^{\prime} C^{\prime}$ as a quartic function of $z$, its quadrinvariant is a function $(x, y)^{8}$, and its cubinvariant a function $(x, y)^{12}$; and similarly, considering $B^{2}-A C$ as a quartic function of $w$, its invariants are functions $(x, y)^{8}$ and $(x, y)^{12}$. We have thus, between the two cones, a geometrical relation answering to the analytical one of the identity of the invariants; but the nature of this geometrical relation is not obvious; and it presents itself as an interesting subject of investigation. 